\title{
Correction to: Lag times to steady state drug delivery by continuous intravenous infusion: direct comparison of peristaltic and syringe pump performance identifies contributions from infusion system dead volume and pump startup characteristics
}

\author{
Lauren E. Gibson $^{1}$ (D) Anders S. Knudsen ${ }^{2} \cdot$ David Arney $^{2} \cdot$ Hao Deng $^{1} \cdot$ Nathaniel M. Sims $^{1} \cdot$ Robert A. Peterfreund $^{1}$
}

Published online: 6 January 2022

(c) Springer Nature B.V. 2022

\section{Correction to: \\ Journal of Clinical Monitoring and Computing https://doi.org/10.1007/s10877-021-00790-1}

In the original publication of the article, the heading 'dark teal' was inadvertently included in Table 1 . The original article has been corrected and the corrected Table 1 is provided here.

The original article can be found online at https://doi.org/10.1007/ s10877-021-00790-1.

Lauren E. Gibson

legibson@partners.org

1 Department of Anesthesia, Critical Care, and Pain Medicine, Massachusetts General Hospital, 55 Fruit Street, Boston, MA, USA

2 MD PnP Program, Massachusetts General Hospital, Boston, MA, USA 
Table 1 Time to overcome the combined effects of startup and dead volume to achieve a fraction (5-95\%) of the intended delivery rate after initiation of a drug infusion

\begin{tabular}{|c|c|c|c|c|c|c|}
\hline \multirow{2}{*}{$\begin{array}{l}\text { Fraction of intended } \\
\text { rate }\end{array}$} & \multicolumn{2}{|l|}{ Mean (minutes) } & \multicolumn{2}{|c|}{ Statistics (Bonferroni corrected) } & \multicolumn{2}{|c|}{ Statistics (uncorrected) } \\
\hline & $\begin{array}{l}\text { Peristaltic pump } \\
(\mathrm{n}=5)\end{array}$ & $\begin{array}{l}\text { Syringe pump } \\
(\mathrm{n}=5)\end{array}$ & $98.3 \% \mathrm{CI}$ & $P$ value & $95 \% \mathrm{CI}$ & $\mathrm{P}$ value \\
\hline \multicolumn{7}{|l|}{ Adult model } \\
\hline $5 \%$ & 2.73 & 5.06 & {$[-4.89,0.23]$} & 0.070 & {$[-4.16,-0.50]$} & 0.023 \\
\hline $50 \%$ & 4.10 & 6.96 & {$[-5.84,0.13]$} & 0.059 & {$[-4.97,-0.74]$} & 0.020 \\
\hline $95 \%$ & 5.69 & 10.37 & {$[-10.11,0.75]$} & 0.083 & {$[-8.54,-0.82]$} & 0.028 \\
\hline \multicolumn{7}{|l|}{ Intermediate model ${ }^{\mathrm{a}}$} \\
\hline $25 \%$ & 17.52 & 15.84 & {$[0.87,2.50]$} & 0.001 & {$[1.07,2.30]$} & $<0.001$ \\
\hline $50 \%$ & 19.13 & 17.41 & {$[0.52,2.92]$} & 0.011 & {$[0.83,2.61]$} & 0.004 \\
\hline $75 \%$ & 23.40 & 19.78 & {$[0.69,6.54]$} & 0.021 & {$[1.44,5.79]$} & 0.007 \\
\hline \multirow{2}{*}{$\begin{array}{l}\text { Fraction of intended } \\
\text { rate }\end{array}$} & \multicolumn{2}{|l|}{ Mean (minutes) } & \multicolumn{2}{|c|}{ Statistics (Bonferroni corrected) } & \multicolumn{2}{|c|}{ Statistics (uncorrected) } \\
\hline & $\begin{array}{l}\text { Peristaltic pump } \\
(\mathrm{n}=8)\end{array}$ & $\begin{array}{l}\text { Syringe pump } \\
(\mathrm{n}=8)\end{array}$ & $98.3 \% \mathrm{CI}$ & $\mathrm{P}$ value & $95 \% \mathrm{CI}$ & $\mathrm{P}$ value \\
\hline \multicolumn{7}{|l|}{ Pediatric model } \\
\hline $5 \%$ & 0.83 & 5.92 & {$[-10.23,0.04]$} & 0.053 & {$[-9.13,-1.05]$} & 0.018 \\
\hline $50 \%$ & 19.11 & 14.91 & {$[2.57,5.84]$} & $<0.001$ & {$[2.90,5.50]$} & $<0.001$ \\
\hline $95 \%$ & 31.49 & 17.99 & {$[6.64,20.35]$} & $<0.001$ & {$[8.19,18.80]$} & $<0.001$ \\
\hline
\end{tabular}

${ }^{\text {a }}$ Because $95 \%$ of the intended drug delivery rate was never achieved in the intermediate model, we report the time required to reach $25 \%, 50 \%$, and $75 \%$ of the intended delivery rate; using $25 \%$ rather than $5 \%$ to maintain symmetry

Publisher's Note Springer Nature remains neutral with regard to jurisdictional claims in published maps and institutional affiliations. 